\title{
Large suprasellar aneurysms imitating pituitary tumour
}

\author{
L. A. R A YMOND A N D J T E W \\ From the Department of Ophthalmology and Division of Neurosurgery, University of Cincinnati \\ Medical Center, and Department of Neurosurgery, Good Samaritan Hospital, Cincinnati, Ohio, USA
}

SUMMARY Two patients had large $(20-30 \mathrm{~mm})$ suprasellar aneurysms with clinical findings resembling an expanding pituitary tumour. One aneurysm was giant $(30 \mathrm{~mm})$. Its delayed diagnosis for six years led to severe chiasmal compression. The other aneurysm was compressing the optic nerve. Visual improvement resulted after surgery.

This report records two cases of large, unruptured suprasellar aneurysms presenting with unilateral visual field loss and a slowly progressive course compatible with a pituitary tumour. They are presented to call attention to this situation which, if unrecognised, can lead to delayed diagnosis and serious errors in management.

An early recorded case of a large carotid aneurysm simulating a pituitary tumour was reported by Mitchell (1889). Postmortem examination revealed an aneurysm $22 \mathrm{~mm}$ (one inch) in diameter embedded in the sella turcica. This aneurysm had "lifted the chiasm until this parted in the middle line, leaving a nerve on each side. . . "Cushing (1912) reported a case with progressive failure of vision and optic atrophy from an hypophyseal adenoma with an interpeduncular aneurysm. He noted that the aneurysm may play the same role as the interpeduncular tumour by its compression effects.

Because both a large intracranial, unruptured aneurysm and an expanding tumour can produce pressure on adjacent structures, it has been suggested that they cannot be distinguished by visual fields (Walsh, 1964). However, in another report (Bird et al., 1970) the pattern of the visual field loss and temporal profile in unruptured suprasellar aneurysms appeared to differ from that of intrasellar or parasellar tumours.

Address for reprint requests: Dr L. A. Raymond, Retinal Service, Department of Ophthalmology, University of Cincinnati Medical Center, Holmes Hospital, Eden and Bethesda Avenues, Cincinnati, Ohio 45219, USA.

Accepted 15 August 1977

\section{Case reports}

CASE 1 LARGE LEFT OPHTHALMIC ARTERY ANEURYSM WITH OPTIC NERVE COMPRESSION

A 53 year old lady had multiple changes in glasses over a period of three years. She was informed in March 1973 by her ophthalmologist that vision was decreased in the left eye. Headaches were infrequent.

She was admitted to Good Samaritan Hospital in Cincinnati, Ohio on 16 January 1974. Positive physical findings were confined to the neuroophthalmological examination. Best visual acuity with correction for the right eye was $20 / 20-1$, and for the left eye $20 / 60+1$. A Marcus Gunn pupil was observed for the left eye. Colour vision testing with the A-O H-R-R pseudoisochromatic plates revealed five out of 15 missed for the right eye and all 15 missed for the left eye. Temporal pallor of the left disc was present. The visual field in the right eye was normal, even with reduced illumination and a red target. The visual field in the left eye revealed a dense loss of the nasal field and a superior temporal defect with larger test objects. With the $3 \mathrm{~mm}$ white target at $1000 \mathrm{~mm}$, a temporal field remained at 5 degrees (Fig. 1). Decreased smelling function was noted on the left side.

Lateral laminograms of the sella turcica showed cortical bony erosion of the proximal portion of the planum sphenoidale. The anterior clinoid process on the left was not sharply defined.

A pneumoencephalogram revealed good filling of the ventricular system and subarachnoid space. Part 1 of the left lateral ventricle showed a suspicious indentation of its under surface. The anterior third ventricle was not well demonstrated. 


\section{LEFT EYE 20/60 RIGHT EYE 20/20}

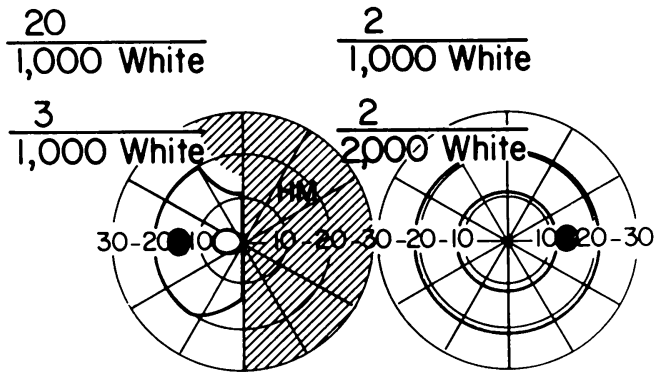

Fig. 1 Preoperative visual fields (case I).

On one tomogram there was a suspicion of some mass effect in the region of the lamina terminalis.

Bilateral carotid arteriograms revealed a large $19 \times 22 \times 20 \mathrm{~mm}$ suprasellar aneurysm (Fig. 2) originating from the left internal carotid artery at the origin of the ophthalmic artery. There was some elevation of both the A-1 segments of the anterior cerebral arteries. The left A-1 segment of the anterior cerebral artery was hypoplastic.

The following studies were normal: electro-

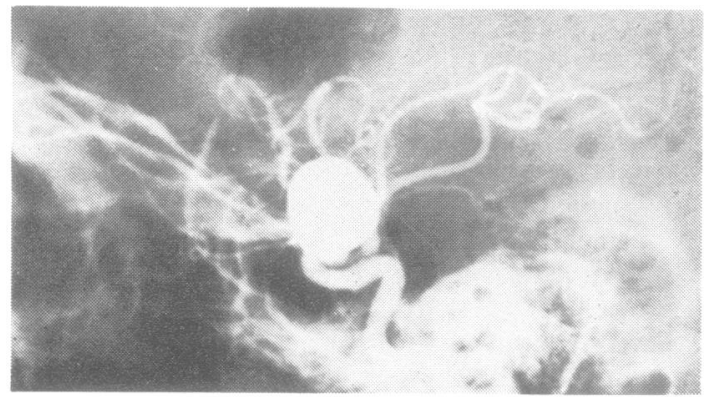

(a)

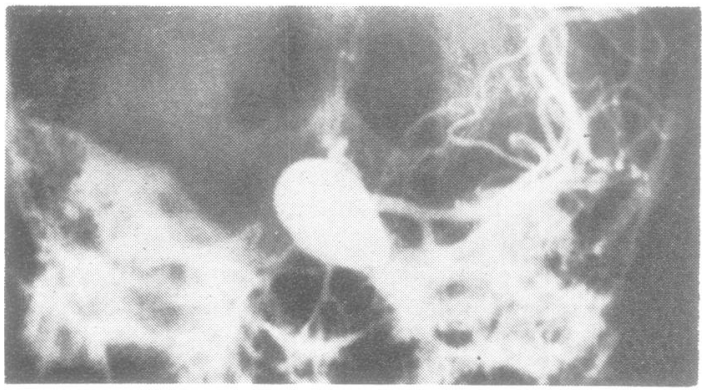

(b)

Fig. 2 Carotid arteriogram showing large ancurysm of ophthalmic artery (case 1) (a) lateral view, (b) anteroposterior view. encephalogram; cerebral flow study and brain scan ("9.9 $\mathrm{m}$ Tc pertechnetate); and examination of the spinal fluid.

On 24 January 1974 a left frontal craniotomy was performed. Temporary occlusion of the left internal carotid artery in the neck was done for control of bleeding at the time of exposure of the ophthalmic artery aneurysm. A large aneurysm of the left ophthalmic artery originated from the internal carotid artery and was underlying the left anterior clinoid process. A small aneurysm of the left posterior cerebral artery, previously observed by angiography, was demonstrated. Both aneurysms were treated by ligation. Excision of the aneurysmal sac of the ophthalmic artery was performed.

On the same day, after the operation, the patient developed right hemiparesis and dysphasia. An arteriogram showed total occlusion of the left internal carotid artery and embolus formation to a branch of the left middle cerebral artery.

Her muscular weakness improved rapidly and $\ddagger$ speech capacity returned. She was discharged on 8 March 1974.

On 20 August 1974 neurological examinatio demonstrated progressive improvement to nearl normal status. Her best visual acuity in the right eye was $20 / 25$ and in the left eye $20 / 30+1$. She now missed only five out of 15 colour plates wit the left eye. The temporal field in the left eye has opened from 5 degrees preoperatively with the $3 \mathrm{~mm}$ white test object to a field essentially fuf $\infty$ temporally with a $2 \mathrm{~mm}$ white test object.

CASE 2 GIANT ANEURYSM OF THE LEFT INTERNAL CAROTID ARTERY ORIGINATING NEAR THE ORIGIN OF THE POSTERIOR COMMUNICATING ARTERY

A 59 year old nun was examined in 1967 by an ophthalmologist for absence of temporal vision in the left eye. Optic neuritis was diagnosed and appropriate treatment given. Subsequent progressive visual loss in the left eye continued to near blindness, and a temporal visual field defect developed in the right eye. In 1972 she noted the onset of difficulty in walking and weakness in the upper extremities.

On 4 January 1973 she was admitted to Good Samaritan Hospital in Cincinnati, Ohio. Physical examination was normal except for neuroophthalmological findings. Visual acuity in the right eye was 20/40-2 and for the left eye counting fingers at $1.2 \mathrm{~m}$ (4 feet). Temporal pallor of the left disc was noted. Bitemporal hemianopsia (Figs 3 and 4) was demonstrated.

A mild right central facial weakness, bilateral spasticity of gait, hyperactive deep tendon reflexes, 


\section{CF 4 feet}

\section{0}

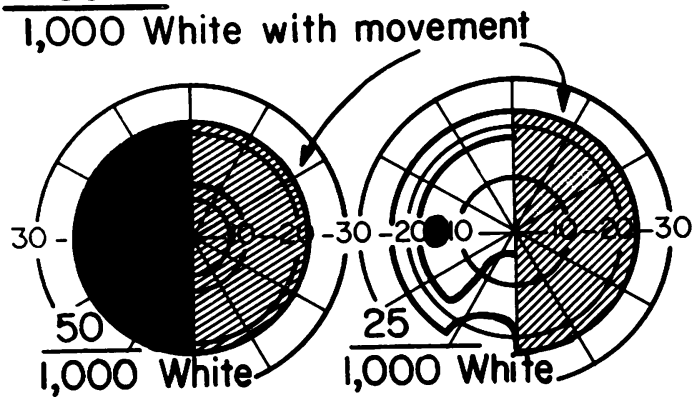

January 1973 October 1973

Fig. 3 Visual fields, left eye (case 2).

$20 / 40$
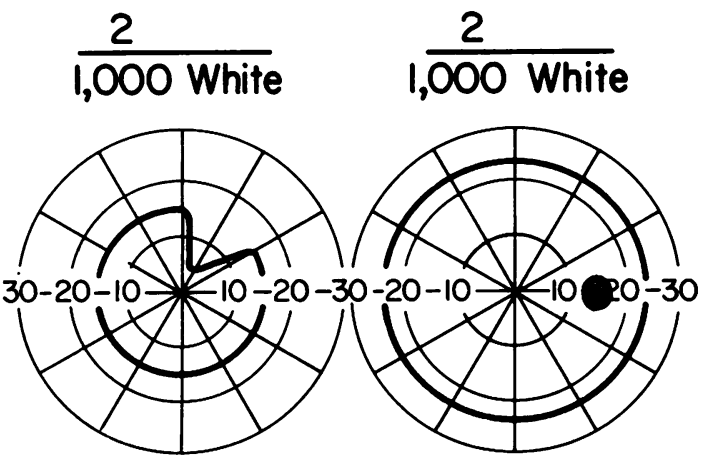

January 1973 October 1973

Fig. 4 Visual fields, right eye (case 2).

and bilateral extensor plantar responses were observed. Slight muscular weakness of extremities was noted, greater on the right than on the left side.

Bilateral cerebral angiography revealed a giant $30 \times 30 \times 30 \mathrm{~mm}$ suprasellar aneurysm (Fig. 5) of the left internal carotid artery and marked distortion of the left frontal lobe by elevation of the left anterior cerebral artery, the anterior communicating artery, and the right anterior cerebral artery. The cavernous portion of each internal carotid artery was displaced laterally. The aneurysm appeared to originate near the origin of the posterior communicating artery and was both intra- and extracavernous. Non-filling of the left ophthalmic artery was observed. The aneurysm

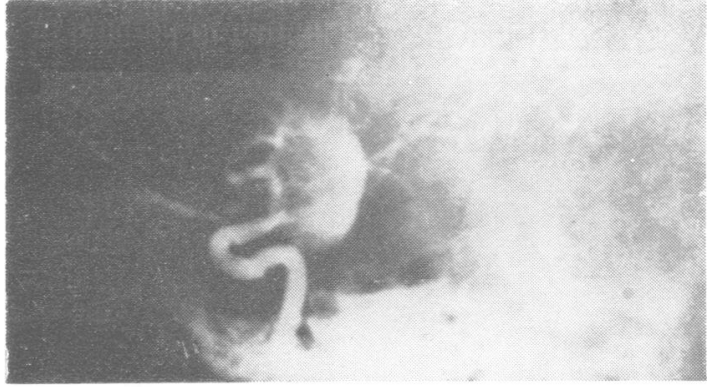

(a)

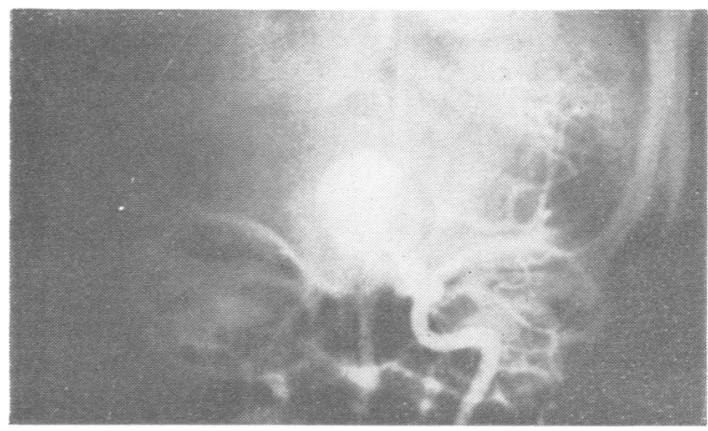

(b)

Fig. 5 Left carotid arteriogram showing giant, midline aneurysm (case 2) (a) lateral view, (b) anteroposterior view.

filled on injection of both internal carotid arteries. Radiographs of the optic foramina, sella turcica, and sphenoidal wing were normal.

On 11 January 1973 the patient underwent ligation of both the internal and external carotid arteries on the left side by a gradual occlusion method with a Crutchfield clamp. On 24 January a right brachial angiogram showed cross-filling from the right to the left carotid artery. On 25 January, through a left frontal craniotomy, the distal portion of the left internal carotid artery was ligated, and this in effect trapped the aneurysm. The large aneurysmal cavity was opened. Clot and fibrin material were removed from its lumen, thereby decompressing the optic nerves (Fig. 6).

The patient's postoperative course was uneventful. She was discharged from hospital on 10 March 1973. Neurological examination in August 1973 demonstrated progressive improvement to nearly normal status.

On 16 October 1973 her best visual acuity with corrections was $20 / 20$ in the right eye and 20/200 in the left eye. The visual field of the right eye was full. The visual field of the left eye demonstrated considerable improvement in the temporal field 


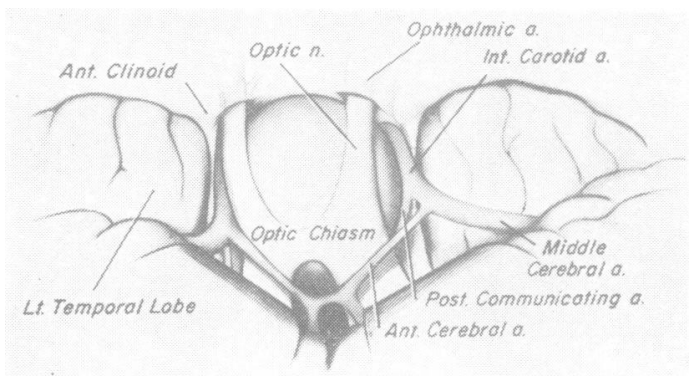

Fig. 6 Artist's drawing of relationship of giant aneurysm to surrounding structures at operation (case 2).

and persistence of the dense nasal field loss (Figs. 3 and 4$)$.

\section{Discussion}

Delayed diagnosis for lesions compressing the chiasm has been attributed to overemphasis of mild macular disturbances, discounting of optic disc pallor, and infrequent measurements or misinterpretations of visual field abnormalities (Pruett and Wepsic, 1973). Delayed diagnosis, extending over several years, occurred in our two cases, one of whom had chiasmal compression. Subtle differences in the disc pallor, easily overlooked by direct ophthalmoscopy, may be better appreciated with simultaneous comparison of stereoscopic coloured photographs of the two optic discs. These changes can be correlated with serial visual fields.

White and Ballantine (1961), in an excellent review of carotid aneurysms, emphasised the necessity for accurate differential diagnosis in these vascular lesions. With few exceptions the aneurysms were indistinguishable from a chromophobe adenoma or craniopharyngioma, either clinically on the basis of a careful history and physical examination by skull radiographs, or even after arteriography, when the aneurysmal sac was thrombosed in three instances.

Berson et al. (1966) reported three of their own cases of large suprasellar aneurysms of the internal carotid artery. Two of these were defined as giant $(20-30 \mathrm{~mm}$ in size), and one as large (almost $20 \mathrm{~mm}$ in size). The most important clinical finding wasthe abnormal visual fields.

The progression of our two cases was similar to a slowly growing pituitary tumour. The first patient demonstrated no temporal field loss in the contralateral eye (Fig. 1). Arteriography (Fig. 2) revealed an unruptured aneurysm of the ophthalmic artery. This particular type of aneurysm (Walsh and Hoyt, 1969), located lateral to the optic nerve, begins with an unilateral central scotoma and then progresses to a nasal field loss on the involved side. It is comparatively rare (Bull, 1962; Hoyt, 1964; Rubenstein et al., 1968; Sengupta et al., 1976).

Our second patient also demonstrated slow, gradual deterioration in vision. Temporal field loss in the worst eye was noted six years before the bitemporal field loss.

Hoyt (1964), in his excellent treatise on the chiasm, describes its blood supply. He notes that "the anastomoses of superficial and intrinsic chiasmal arterioles is so diffuse that obliteration of single feeder vessels will not cause infarction in the chiasm". Areas of the chiasm that survive compression from extrinsic tumour pressure do so because they have not been subjected to stress sufficient to collapse their capillary network. He, like others (Jefferson, 1937, 1955; Traquair, 1928), cannot relate the resulting field defect from chiasmal damage by extrinsic tumour pressure to the retinotopic fibre relations of the undistorted chiasm.

Angiography is almost always diagnostic pro$v$ :ded the aneurysmal sac is not toially thrombosed. Occasionally the size of the aneurysm is misleading because laminations of clot within the aneurysm may greatly reduce the visible lumen on angiography (Lombardi et al., 1963).

Ligation of the internal carotid artery in the neck is the recommended treatment of unruptured aneurysm, provided the patient's cerebrovascular? system is capable of supplying the necessary crossflow from the contralateral carotid artery (Jefferson, 1937; White and Ballantine, 1961). Evacuation of the clot from the aneurysmal sac may occasionally be necessary to decompress the optic nerves and chiasm, particularly when the diagnosis is made before irreversible optic atrophy has occurred.

\section{References}

Berson, E.. Freeman, M. I., and Gay, A. J. (1966). Visual field clefects in giant suprasellar aneurysms of internal carotid. Archives of Ophthalmology, 76, 52-58.

Bird, A. C.. Nolan, B., Gargano, F. P., and David, N. J. (1970). Unruptured aneurysm of the supraclinoid carotid artery - a treatable cause of blindness. Neurology (Minneapolis), 20, 445-454.

Bull, J. W. D. (1962). Contribution of radiology to the study of intracranial aneurysms. British Medical Journal. 2, 1701-1708.

Cushing, H. (1912). The Pituitary Body and Its Disorders: Clinical States produced by Disorders of the Hypophysis Cerebri. pp. 97-99. J. B. Lippincott: Philaclelphia. 
Hoyt, W. F. (1964). The human optic chiasm: a neuroanatomical review of current concepts, recent investigations, and unsolved problems. The University of Miami Neuro-Ophthalmology Symposium Edited by J. L. Smith, pp. 64-111. Charles C. Thomas: Springfield. Illinois.

Jefferson, G. (1937). Compression of the chiasma. optic nerves, and optic tracts by intracranial aneurysms. Brain, 60, 444-497.

Jefferson, G. (1955). Further remarks concerning compression of the optic pathways by intracranial aneurysms. Proceedings of the Congress of Neurological Surgeons, 1, 55-103.

Lombardi, G., Passerini, A., and Migliavacca, F. (1963). Intracavernous aneurysms of the internal carotid artery. American Journal of Roentgenology, Radium Therapy, and Nuclear Medicine, 89, 361371.

Mitchell, S. W. (1889). Aneurysm of an anomalous artery causing antero-posterior division of the chiasm of the optic nerves and producing bitemporal hemianopsia. Journal of Nervous and Mental Disease, 14, 14-62.
Pruett, R., and Wepsic, J. G. (1973). Delayed diagnosis of chiasmal compression. American Journal of Ophthalmology. 76, 229-236.

Rubenstein, M. K., Wilson, G., and Levin, D. C. (1968). Intraorbital aneurysms of the ophthalmic artery-report of a unique case and review of the literature. Archives of Ophthalmology, 80, 42-44.

Sengupta, R. P., Gryspeerdt, G. L., and Hankinson, J. (1976). Carotid-ophthalmic aneurysms. Journal of Neurology, Neurosurgery, and Psychiatry, 39, 837853.

Traquair, H. M. (1928). An Introduction to Clinical Perimetry. p. 189. C. V. Mosby Company: St Louis.

Walsh. F. B. (1964). Visual field defects due to aneurysms at the circle of Willis. Archives of Ophthalmology, 71, 15-27.

Walsh, F. B.. and Hoyt, W.F. (1969). Clinical NeuroOphthalmology, Volume II, third edition, pp. 17481751. Williams and Wilkins: Baltimore.

White, J. C., and Ballantine. H. (1961). Intrasellar aneurysms simulating hypophyseal tumors. Journal of Neurosurgery, 18, 34-50. 